\title{
Measurements of Absolute X-ray Generation Efficiency
}

\author{
M.Satya Prasad* and D. C. Joy ${ }^{* *}$ \\ *EM Facility, University of Tennessee, Knoxville, TN 37996-0810 \\ ** and Oak Ridge National Laboratory, Oak Ridge, TN 37831-6064
}

The rate of generation of X-rays from an element irradiated at some energy E depends on the product of the ionization cross-section $\sigma(\mathrm{E})$ and the fluorescent yield $\omega$. Unfortunately neither of these quantities is well established independently, especially outside of the k-series of lines ${ }^{[1]}$. We have therefore set out to directly measure and parameterize the absolute X-ray generation efficiencies (photons /electron) for a wide range of K,L, and $\mathrm{M}$ lines from different elements. We anticipate that a complete set of such data would be of great value in applications such as spectrum simulation and standardless analysis.

The measurements and the subsequent analysis of the data are a development of the approach first described by Lifshin et al. ${ }^{[2]}$. The experiment consists of a measurement of the emission rate in counts per nanoampere of beam current per second. The majority of the elements examined were part of a microprobe standards block, which are highly polished flat surfaces. Measurements were made for live-time period of $100 \mathrm{~s}$ and several such measurements were averaged at each energy. The beam current was measured immediately before and after each run using a Keithley 414A picoammeter and was adjusted over the range $80 \mathrm{pA}$ to $1.2 \mathrm{nA}$ to maintain an approximately constant count rate with energy. This current was enough to generate 20,000 - 100,000 counts for each peak to ensure good statistics. The net intensity of the chosen X-ray line was obtained by using a linear background substract method. Figure (1) shows a typical experimental curve for Sn L-line vs energy. The measured yields in counts per ampere per second were corrected for self-absorption by using $\mathrm{f}(\chi)$ values that were computed from a Monte Carlo simulation, for the take-off angle of $\theta=35^{\circ}{ }^{[3]}$. The observed count rate is also affected by the efficiency of the EDS detector which in turn depends on the solid angle subtended by the detector at the specimen and by transmission efficiency of the window in front of the detector diode.

The detector quantum efficiency was obtained by comparing the measured yield for the $\mathrm{Cu} \mathrm{K} \alpha$ line at $18 \mathrm{keV}$ with an average of published values for the efficiency of $\mathrm{Cu} \mathrm{K} \alpha$ production at an overvoltage ratio $\mathrm{U}=2(18 \mathrm{keV})$, and was found to be 0.0097 . In order to find the window transmission, the manufacturer's default values have been iteratively refined by using the DTSA spectrum simulation tool to match an experimental carbon continuum spectrum over the energy range $500 \mathrm{eV}$ to $20 \mathrm{keV}$. By knowing the efficiency of the EDS detector and the necessary $f(\chi)$ correction, the measured yield in counts per ampere per second can be corrected to give $\mathrm{N} / 4 \pi$ - the generation efficiency of X-rays within the target in photons per steradian per electron ${ }^{[4]}$. As first shown by Compton \& Allison the magnitude of $\mathrm{N} / 4 \pi$ for K-lines has a functional variation with the overvoltage $U=E / E_{\text {crit }}$ ( where $E$ is the electron beam energy and $E_{c r i t}$ is the critical excitation energy for the line ) of the form ${ }^{[5]}$

$$
\mathrm{N} / 4 \pi=\mathrm{N}_{0} / 4 \pi \cdot(\mathrm{U}-1)^{\mathrm{n}}=A \cdot(\mathrm{U}-1)^{\mathrm{n}}
$$

The measured data are therefore plotted ( Fig. 2) on the logarithmic axes as a function of (U - 1). It can be seen that the agreement between the proposed power law behaviour and the experimental data 
is generally good. The absolute X-ray generation efficiency as a function of (U-1) for the K-lines of eight elements, for the L-lines of 17 elements, and for the M-lines of six elements were plotted and fit to A.(U-1) ${ }^{\mathrm{n}}$ expression. We find that equation (1) applies with good accuracy to K, L, and M lines over a wide range of $U$ values. Work is now in progress to parameterize the values of $A$ and $n$ as a function of atomic number, line excited, and the overvoltage.

\section{References}

[1] For a complete listing of experimental $\sigma$ and $\omega$ values see web.utk.edu/ srcutk.

[2] E. Lifshin et al., Proc. $8^{\text {th }}$ IXCOM, Boston 1977,pp141-148 (1980).

[3]D.C. Joy (1995) Monte Carlo Modeling for Electron Microscopy and Microanalysis, Oxford University Pres, New York, 174-198.

[4]D.C. Joy , Journal of Microscopy. Vol.191. Pt 1. July 1998. pp. 74-82.

[5]A.H.Compton \& S.K.Allison (1935)X-rays in Theory and Experiment, Van Nostrand, New York.

[6]This work was partially supported by SRC under contract LJ-413.003. The contract monitor is Dr.D.Herr.

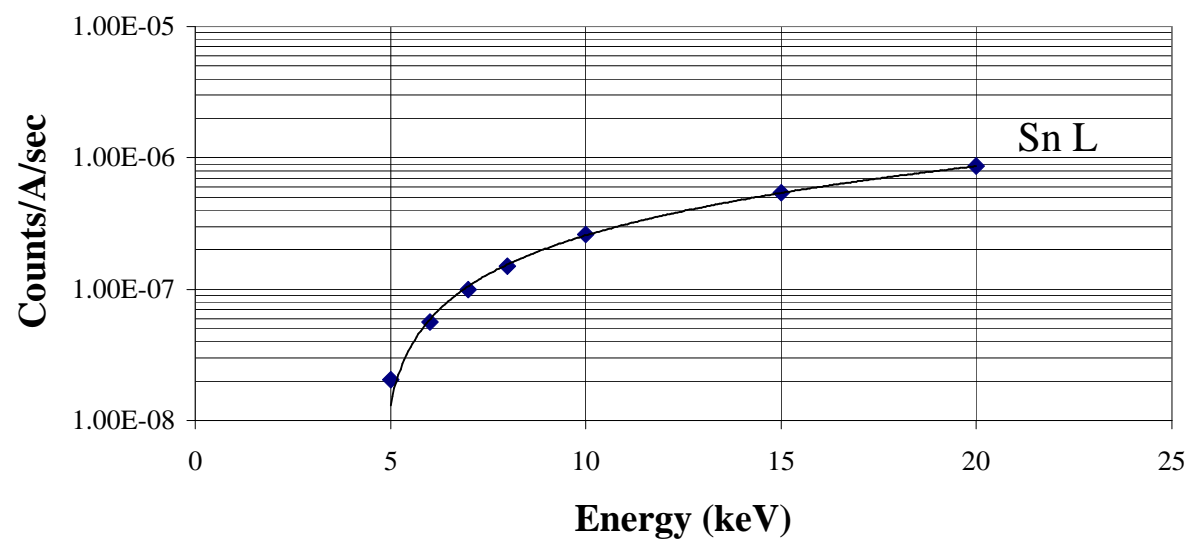

FIG. 1. Variation of count rate with beam energy for the Sn L-line.

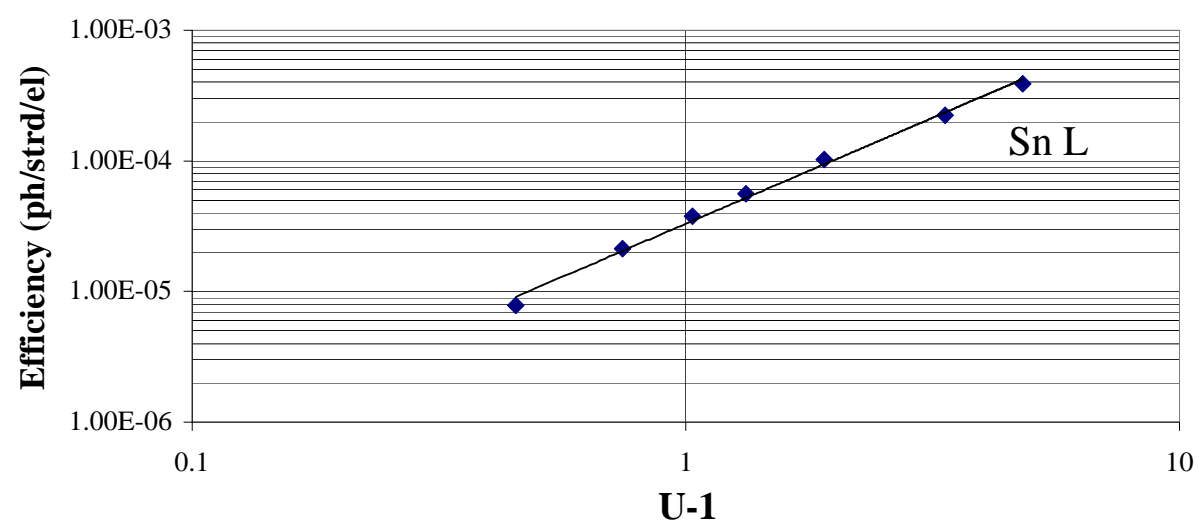

FIG. 2. Variation of absolute $X$-ray generation efficiency as a function of (U-1) for the Sn L-line. 